\title{
Scientific Culture, Power and Sustainability in Environmental Intervention Schemes
}

\author{
J. Andrés Domínguez-Gómez and Fernando Relinque-Medina
}

\begin{abstract}
The lack of real integration between the different dimensions of sustainability is highlighted in the literature as one of the main hindrances to lessening the environmental risks and impacts of large-scale development projects. This paper discusses a hypothesis concerning the problems of integration between the different dimensions of sustainability in socio-environmental research into such projects. Differences in modes of knowledge production and the relationships of these with prevailing social positions constitute the crux of the argument. Endeavours to improve translational knowledge and mixed research methodologies (seeking the semi-standardization of research processes) are put forward as approaches to overcoming the above-mentioned obstacles.
\end{abstract}

Index Terms-Socio-environmental impact assessment, sustainability, translational knowledge.

\section{ISSUES IN SOCIO-ENVIRONMENTAL INTEGRATION, DifFERENCES IN SCIENTIFIC CULTURES AND PREVAILING SOCIAL POSITIONS}

It is now many years since the concept of "sustainable development" was formulated. It is an idea that has been debated and deployed extremely widely, broadened in composition and definition, and nuanced and split into a range of conceptual sub-products (for example sustainable local development) [1]. Since its formulation, the need to integrate its different dimensions has been referred to possibly millions of times: the need for the simultaneous consideration of these dimensions, in order to achieve the probably utopian condition in which all societies can grow socioeconomically without compromising the right and/or need of future generations to enjoy their environment to the same extent and in the same conditions as current generations. These millions of references have issued from every information- and knowledge-producing body, from the scientific sector to businesses, including politics, civil society, the media, etc.

Despite the topic's long history, today we are witnessing a deep and bewildering deficit in the real integration of the different dimensions of sustainability. While on the theoretical-conceptual level the need for integration is widely and perfectly recognized and detailed, it is difficult to find actual development projects whose design and/or application integrate these various dimensions. Many analysts concur

Manuscript received June 3, 2016; revised December 23, 2016. This research has been founded by Ministry of Economy, Spanish Government (30\%, code CSO2012-32493), and Innovation Department of Andalusian Government (Spain) (70\%, code SEJ2397) within the context of the so called project "Methodology for social impact assessment of golf courses".

The authors are with University of Huelva, Spain (e-mail: andres.dominguez@eismethods.com,fernando.relinque@pi.uhu.es). that this lack of real integration is the most cogent explanation for the continued advance of climate change, our main contemporary threat [2].

Large-scale development projects (LDP from here on: for example infrastructure and transport development, power supply networks, hydroelectric dams, industrial and mining projects, urban plans and developments, etc.) form a field in which this issue is fundamental. The specialized literature constantly stresses the need for multidimensional integration, in addition to the transdisciplinarity of the field's object and the interdisciplinarity of its approach [3] [4]; always in ideal terms, however, since the difficulty of this integration and, finally, the resulting deficits in the sustainability of development projects, are also always underlined.

One of the most-used tools for improving sustainability in LDP is environmental impact assessment (EIA from here on). This took off during the global environmental crisis of the 1970s and has spread worldwide, driven by the expansion of ecological culture and especially the regulatory pressure for all LDP to foresee the environmental risks incurred by each scheme. EIA studies include, in general, a phase in which the project's social risks and impacts ("social impact assessment," SIA from here on) are accounted for [5]. Global regulatory trends, set by the countries which are more advanced in and sensitized to environmental issues (USA, Canada and Australia for example) [6], have gradually recognized the need to investigate and assess social aspects in addition to strictly environmental ones.

Thus, on paper, studies prior to the carrying out of LDP already envisage the inclusion of the different dimensions of the environmental spectrum. Each administrative body with power to legislate (national governments, normally, or international ones, the EU for example) officially recognizes that preliminary studies for an LDP of any scope should account for risks and impacts on the environmental, social and economic levels [7]. This last, of course, is integrated "by default" by developers in their preliminary studies, since without economic profits in the short, medium and/or long term, no developer would initiate an LDP. Unfortunately, this multiplicity of dimensions, although widely recognized, is rarely put into practice [8]-[10].

In this paper we attempt to broaden analyses from previous studies [11], [12] in which the theoretical, methodological, epistemological, ethical and ontological bases of SIA studies were systematized. The studies cited refer to what "should be," i.e. the ideal foundations for the design, carrying out and functioning of SIA studies. Here we attempt to add some key strategic points which may enable applied science (both environmental and social) to advance towards sustainable LDP. We bring to the debate a specific explanation of the problems of integration in socio-environmental research into 
LDP. Firstly we outline the two different forms of knowledge production [13] corresponding to the scientific cultures of the natural and engineering sciences and the social sciences. We observe that decision-makers regard only one of these forms as "appropriate" or legitimate, discriminating against the other as being difficult to measure due to the complexity of its object (and therefore also difficult to integrate into the socially legitimated model of knowledge), and also because its approach is markedly alternative to the norm [14], [15]. These key points are also strategic for applied social sciences, since the inclusion of its outputs in research into LDP would doubtless contribute to boosting the social-scientific disciplines, in both the academic and professional fields.

The design and management of development projects is a field of applied science practically monopolized by the natural sciences: fields such as geology, climatology, hydrology, engineering of all kinds, etc.; not forgetting economics, closer to statistical methods of financial risk calculation and modelling. Political bodies (policy-makers) and other decision-makers involved in LDP usually place their trust in these sciences and make decisions based on the results yielded by these traditional methods [16], [17]. In other words, decisions on the design and implementation of projects are taken according to a mode of knowledge production seen as "scientific," and which is generalized, accepted and legitimated among specialized consulting professionals [18].

Further, decision-makers hold dominant political positions relative to other stakeholders involved in the context of each project, to the point of imposing, if necessary, their interests over those of other actors. They are the influential actors, who share objectives (e.g. that projects are carried out and made operational as quickly as possible) and discourses (e.g. that the project is good for the area). For example, in urban/tourist developments and hydroelectric dams we have found discursive communities bringing together policy-makers, local administrations and developers. These actors' arguments are in favour of projects and stress the great economic and environmental benefits that they bring to a specific geographical locale. As against this type of argument we also find a different and more critical discourse deriving from other local stakeholders (the population of the area, small local businesses, civil society, etc.; i.e. the least influential actors in the design and implementation of the LDP). These less influential actors' main argument is exactly the contrary: that such projects bring hardly any kind of benefit to the local community, the most important being economic profits for the developers (who are often from outside the area) and the political profitability of the initiative (for the political actors). The negative impacts, however, are borne by these least influential actors, thus creating serious environmental and ethical deficits for the LDP, and therefore for the sustainability of the initiative [19].

Therefore we identify two obstacles to the integration of environmental dimensions in LDP preliminary studies. (a) The first obstacle relates to the cultural clash between scientific paradigms, in which the stronger position is held by the traditional paradigm ("mode 1" of knowledge production in Gibbons' terminology [13]. (b) The second obstacle stems from the trust placed by the dominant stakeholders (decision-makers and developers) in this scientific practice. These two obstacles are related to each other. Basically it is a question of different ways of understanding and interpreting reality: the positivist paradigm as opposed to the constructivist, the first seeking knowledge through analysis (decomposition), modelling and simplification, and the second through interrelation, induction and interpretation. The concurrence of the most influential actors with the positivist paradigm adds another dimension to the debate. Powerful social actors (those who can influence the design and/or implementation of LDP) are, firstly, foreign to the cultural-scientific register of the constructivist paradigm; further, the greater socio-environmental complexity of this paradigm means making more complex, multidimensional and multimethod studies, in order to fully understand (i.e. in sufficient depth and detail) the risks and/or impacts of an LDP. Dealing with this complexity, and acting in accordance with it, would mean delaying, and on occasion calling into question, the main objective of the LDP's influential actors: to design and implement development projects [20]-[22]. These obstacles explain, at least in part, the great difficulties that social scientists who specialize in complex, dynamic and context-dependent objects of study face in getting access to professional work (namely, project consultancy). Each obstacle is a challenge, both scientific and professional, to the community of SIA analysts and practitioners, and the final result is that projects are challenged in their sustainability.

\section{The Key Role OF TRANSLATiOnAL KNOWLEDGE}

Overcoming these challenges means bringing together the cultures of the two paradigms by means of translational knowledge [23], a concept which refers to the need to create effective communication between areas of knowledge or practice currently distanced from each other. This distance is reflected in a lack of communication and mutual understanding resulting in the loss of opportunities on both sides [24]. Translation knowledge is more widely accepted in fields where cultural difference is a key element in problem solving, for example in preventive medicine [25], where lifestyle habits and socio-environmental relationships are as important or more so than the medical treatments themselves, and the cultural distance between both sides impacts on the effectiveness and efficacy of treatments and public health in general. This is also the case in the transfer of knowledge and technology between universities (especially in the south of Europe) and their productive surroundings (the market and civil society) [26]. Society's lack of knowledge about university activities, especially in applied research; the universities' lack of proactivity in spreading knowledge; cultural stereotypes which see the university as a solemn, brainy institution which "does important things" but "no one knows exactly what," etc., are factors highlighting the need for translation knowledge to bridge the gap in order to benefit from relational synergies and boost the achievement of institutional objectives (preventing illness and improving public health, or in the second case raising levels of social and technological innovation and developing the universities' social role) [27].

Thus there is seen to be a pressing need for translation 
knowledge to bring together the natural sciences and the social sciences. The historical, theoretical and practical distance between the two spheres is very great, to the point of diverging in paradigmatic terms (the two paradigms of scientific production cited above); and this is the main barrier to their integration into a science which, applied to a sustainability, would be both efficacious and efficient, resulting in development projects that would also be truly sustainable, in the fullest sense of the word. Further, the fact that the natural sciences, which share the positivist paradigm, hold privileged social positions, makes the building of translation knowledge still more difficult. Such privileged positions are seen in society's view of science (i.e., what is understood by people as "science"), reflecting cultural stereotypes, and in this science's close relationship with decision-making bodies. In other words, the social actors with the greatest power to influence and modify the environment, as well as subscribing to these stereotypes, seek knowledge from and place their trust in the natural sciences, which are thereby socially legitimized to work in the area of relationships with the environment.

\section{The Key Role OF SEMI-STANDARDIZATION (IN SEARCH OF PARSIMONY)}

The social sciences could be more proactive in the creation of this translational knowledge aiming to bridge the gap between paradigms. Mixed methodologies, as cultural and communicative areas which blend traditional-positivist sciences with constructivist-social, could serve as methodological bridges, facilitating understanding by and integration into the positivist paradigm, or at least making the social-science paradigm more approachable to natural scientists. Such methodologies could also allow social scientists to approach the privileged positions of the natural sciences and their relationships with decision-makers. In the framework of LDP preliminary studies (EIA and SIA, essentially), the semi-standardization of technical-methodological procedures could also be a key strategy. Establishing a clearly laid-out series of steps or stages for EIS, with intermediate outcomes (results of analyses) which would feed into subsequent stages; predefining the different dimensions to embrace (and their related indicators); determining the most frequent stakeholders according to the type and phase of the project, etc., would be some of the parts and processes of EIS which could be semi-standardized, based principally on expert criteria supported by the literature (specialized bibliography) and prior experience [28]-[30].

One of the principal criticisms of SIA studies is the high level of complexity and the relativism of their results, to a large extent consistent with the study of highly complex and dynamic objects [31]-[33]. This means that most SIA remain case studies, whose usefulness for their client projects depends on the social engagement or responsibility of the developer and the strictness of the regulations applied to the project. A cursory bibliographical analysis of EIS studies [34] shows a major increase in production in the last 25 years (93\% of papers were published between 1990 and April 2014), and a wide diversity of subject matter (energy projects -electrical power networks, dams, wind farms, etc-agricultural projects, forest management, industry, mining, tourism, transport infrastructures, waste disposal, urban development). But it also reveals clear evidence of a relativist and localist approach, with $63 \%$ of all studies centring on specific cases of risk/impact assessment. On the other hand, $37 \%$ were broader analyses, or moved in the realm of general theory or methodology, with the methodological "mid-range" noticeably lacking. Thus there was a remarkable absence of studies which, basing themselves on constantly accumulating worldwide experience in SIA, set out organized stages and internal processes which could be taken as models for further studies. In other words, there is a lack of studies advancing towards the semi-standardization of SIA stages and intermediate outcomes, and going further than the details and particularities of each case, although the discipline is currently able to draw on the experience of practitioners from all over the world and in every type of development scheme. Esteves' full and more recent literature review [35] is consistent with the data cited above, and also demonstrates the strategic interest of standardization of processes and indicators for developing corporate social responsibility in the institutional and business worlds.

Despite the scarce bibliography in this mid-range of the SIA field, in the wider area of socio-environmental studies there are more examples of this type of methodological research oriented towards measurement in complex contexts. We could cite for example methodologies such as the multi-agent simulation model [36], or models based on diffuse logic [37], as well as the design of scenarios using multimethod techniques [38], along with assessment "for" sustainability [rather than assessment "of" sustainability] [39] and participatory integrated assessment [40]. There are also systems of indicators systematized by dimensions, like that of ecosystems services [41], with its roots in classic systems theory [42], and systems of indicators for the most difficult-to-measure features of an environmental complex [43]. These methodologies or conceptual systematizations have in common the assumption of complexity as the field of play in each piece of research, in addition to the search for parsimonious models which can strike a balance between prediction and efficiency [44]: models which both explain reality and, without laying claim to an unattainable total explanation of the processes and phenomena analysed, provide outputs of a scientific quality acceptable for decision making.

The principle of parsimony adopted by these models affords strategic advantages for SIA and, in turn, for LDP. All of them feature an approximation of mode 1 methodologies to the research contexts for which mode 2 is shown to be more appropriate. Thus we may advance towards a methodological mid-point, bringing scientific subcultures closer to each other and improving communication between the two spheres, the traditional positive sphere (found in engineering and natural sciences) and the contextualized constructivist sphere (more habitual in the social sciences). At the same time these methodological bridges bring political positions closer, between those who monopolize professional practice in consulting for decision-making (basically engineering, the normal model 
for policy- and decision-makers), and the social scientists, less favoured but increasingly important in real risk control in complex contexts. This political rapprochement is facilitated by the appearance on the international business scene of ideas such as Corporate Social Responsibility, and, in the extracting sector, the Social Operating License [45], [46], both clear examples of the growing importance of ethical and social issues in development projects.

Thus this principle of semi-standardization should contribute to the integration of social studies into the ensemble of studies stemming from a project, to the degree that, firstly, the resulting methodologies adopt approaches to their object similar to those of engineering, while, secondly, results are communicated, interpreted and (ideally) integrated into those obtained from the other dimensions of risk/impact studies. Finally, the adoption of this principle should also contribute to improving project sustainability, since methodological and political rapprochement would result in better control of risks in terms of both quantity (the size of the risks) and quality (control of a greater diversity of risks).

\section{CONCLUSION}

The specialized literature stresses the need to integrate the various dimensions of the environment into the design and implementation of LDP. This is seen as an indispensable requirement for profitable, ethically acceptable projects that would respect both the social and natural environments. Ignoring any one of these dimensions of the environment, from the preliminary studies stage (as in EIA and SIA) to the dismantling of facilities and cessation of activity, generates risks and impacts which are often difficult to control, and which may even cause wholesale failure of the project.

This paper argues that cultural differences within the scientific community are related to the different modes of knowledge production. One of these modes, apart from being socially legitimized, is common to social actors who exert influence on decision-making in the planning and implementation of LDP; it is the mode corresponding to science in the traditional sense, i.e. the natural sciences and their applications. The second responds to the needs of a highly complex contemporary society which generates socio-environmental contexts needing to be studied and understood in multidimensional and interrelational ways; and this is the scientific culture of social research.

Two obstacles emerge, then: the cultural and the political (i.e. relating to the positions of power occupied by the different areas of knowledge in the professional marketplace); and in order to overcome these obstacles we suggest that translational knowledge should be promoted, along with a mixed methodological language, as a parsimonious mode of bringing together positivist and constructivist standpoints. We hope that improvements in mutual understanding and acceptance between the two positions will create more realistic, contextualized and positive knowledge and technologies for reducing the socio-environmental risks and impacts of LDP.

\section{REFERENCES}

[1] D. C. Gibbs, J. Longhurst, and C. Braithwaite, "Struggling with sustainability," Weak and Strong Interpretations of Sustainable Development within Local Authority Policy, 1998, vol. 30, no. 8, pp. 1351-65.

[2] U. Schubert and E. Störmer, "Sustainable development in Europe?: Concepts, evaluation, and applications," Cheltenham: Edward Elga, 2006, p. 340.

[3] E. João, F. Vanclay, and L. Broeder, "Emphasising enhancement in all forms of impact assessment: Introduction to a special issue," Impact Assess Proj Apprais, vol. 29, no. 3, pp. 170-80, 2011.

[4] F. Vanclay and D. A. Bronstein, Environmental and Social Impact Assessment, John Wiley \& Sons, 1995.

[5] J. A. Pisani and L. A. Sandham, "Assessing the performance of SIA in the EIA context: A case study of South Africa," Environ Impact Assess Rev., 2006, vol. 26, no. 8, pp. 707-24.

[6] J. Asselin and J. R. Parkins, "Comparative case study as social impact assessment: Possibilities and limitations for anticipating social change in the far North," Soc Indic Res., 2009, vol. 94, no. 3, pp. 483-97.

[7] C. Wood, Environmental Impact Assessment: A Comparative Review, Longman: Harlow, 1995.

[8] R. Jha and K. Murthy, "A critique of the environmental sustainability index," SSRN Work Pap Ser, 2003.

[9] R. G. Feeney, "Evaluating the use of social impact assessment in Northeast US federal fisheries management," Impact Assess Proj Apprais., 2013, vol. 31, no. 4, pp. 271-279.

[10] M. Pasgaard, "The challenge of assessing social dimensions of avoided deforestation: Examples from Cambodia," Environ Impact Assess Rev., 2013, vol. 38, pp. 64-72.

[11] J. A. Domínguez-Gómez, "Four conceptual issues to consider in integrating social and environmental factors in risk and impact assessments," Environ Impact Assess Rev., 2016, vol. 56, pp. 113-119.

[12] A. Aledo-Tur and J. A. Domínguez-Gómez, "Social impact assessment from a multidimensional paradigmatic perspective: Challenges and opportunities," J. Environ Manage.

[13] M. Gibbons, "The new production of knowledge: The dynamics of science and research in contemporary societies," SAGE, 1994, p. 196.

[14] H. Nowotny et al., "Re-thinking science," Re-Think Sci Knowl Public Age Uncertain, 2001.

[15] B. Flybjerg, Real Social Science Applied Phronesis, Cambridge University Press, 2012

[16] M. Castells et al., The Cultures of Economic Crisis, Oxford University Press, 2012.

[17] A. Touraine, After the Crisis, Wiley, 2014.

[18] J. Torriti, "The unsustainable rationality of impact assessment," Eur J Law Econ., 2011, vol. 31, no. 3, pp. 307-20.

[19] J. A. Domínguez-Gómez and A. Vargas-Sánchez, "Discussing the socio-economic impacts of tourism development projects based on golf course: The perspective of local stakeholders," Sustainable Tourism, Valencia: WIT Press, 2016.

[20] R. Duncan and P. Hay, "A question of balance in integrated impact assessment: Negotiating away the environmental interest in Australia's Basslink project," J. Environ Assess Policy Manag., 2007, vol. 9, no. 3, pp. 273-97.

[21] B. Flybjerg, "Projects, power, and politics: A Conversation with Bent Flyvbjerg," Proj Power Polit Conversat Bent Flyv-Interview Conduct Jesper Pagh Malene Freudendal-Pedersen Twentyfirst, 2014, vol. 3, pp. 62-75.

[22] B. Flyvbjerg, Rationality and Power: Democracy in Practice, 1st Ed. Chicago: University of Chicago Press; 1998, p. 304.

[23] E. Cremades et al., "Managerial practices driving knowledge creation, learning and transfer in translational research: An exploratory case study," R Manag., 2015, vol. 45, no. 4, pp. 361-85.

[24] P. W. Graham et al., "What is the role of culture, diversity, and community engagement in transdisciplinary translational science?" Transl Behav Med., 2016, vol. 6, no. 1, pp. 115-24.

[25] J. E. C. Filho et al., "The importance of spreading knowledge relating to translational research," Rev Bras Saude Materno Infant., 2013, vol. 13, no. 4, pp. 295-6.

[26] M. Wedgwood, "Mainstreaming the third stream," Mass High Educ., 2005, pp. 134-57.

[27] D. Khodyakov et al., "On using ethical principles of community-engaged research in translational science," Transl Res., 2016, vol. 171, pp. 52-62.

[28] M. Khosa, "11 social impact assessment of development projects," Infrastructure Mandates for Change 1994-1999, Pretoria, South Africa: Human Sciences Research Council, 2000.

[29] M. Consiglio et al., "A guide to social impact assessment in the oil and gas industry," in Proc. 8th SPE International Conference on Health, 
Safety and Environment in Oil and Gas Exploration and Production 2006, pp. 390-395.

[30] V.-A. Cloquell-Ballester et al., "Indicators validation for the improvement of environmental and social impact quantitative assessment," Environ Impact Assess Rev., 2006, vol. 26, no. 1, pp. 79-105.

[31] D. Detombe et al., International Handbook of Social Impact Assessment Conceptual and Methodological Advances, 2003.

[32] P. Stewart, "Complexity theories, social theory, and the question of social complexity," Philos Soc Sci., 2001 Sep., vol. 31, no. 3, pp. 323-360.

[33] N. Stehr and R. Grundmann, "The authority of complexity," $B r J$. Sociol, 2001, vol. 52, no. 2, pp. 313-329.

[34] J. A. Domínguez-Gómez et al., "Social impact assessment of internention schemes on environment: Descriptive analysis of bibliography," Czech Republic, 2017.

[35] A. M. Esteves et al., "Social impact assessment: The state of the art," Impact Assess Proj Apprais, 2012, vol. 30, no. 1, pp. 35-44.

[36] M. A. Janssen and E. Ostrom, "Empirically based, agent-based models," Ecol Soc., 2006, vol. 11, no. 2, pp. 643-55.

[37] C. C. Ragin, Fuzzy-Set Social Science, University of Chicago Press, 2000, p. 374

[38] F. H. Diniz, "From space and from the ground: Determining forest dynamics in settlement projects in the Brazilian amazon," Int For Rev. 2013, vol. 15, no. 4, pp. 442-55.

[39] B. Kommadath et al., "A fuzzy logic based approach to assess sustainable development of the mining and minerals sector," Sustain Dev., 2012, vol. 20, no. 6, pp. 386-99.

[40] P. Harrison et al., "Combining qualitative and quantitative understanding for exploring cross-sectoral climate change impacts, adaptation and vulnerability in Europe," Regional Environmental Change, vol. 13, no. 4.

[41] J. Baker et al., "Ecosystem services in environmental assessment-Help or hindrance?" Environ Impact Assess Rev., 2013, vol. 40, pp. 3-13.

[42] L. Bertalanffy "Perspectives on general system theory: Scientific-philosophical studies," G. Braziller, 1975, p. 190.

[43] Collantonio, A. Social Sustainability: An Exploratory Analysis of its Definition, Assessment Methods, Metrics and Tools, 2007.
[44] T. Snell and R. Cowell, "Scoping in environmental impact assessment: Balancing precaution and efficiency?" Environ Impact Assess Rev. 2006, vol. 26, no. 4, pp. 359-76.

[45] H. Jenkins, "Corporate social responsibility and the mining industry: Conflicts and constructs," Corp Soc Responsib Environ Manag., 2004 vol. 11, no. 1, pp. 23-34.

[46] J. L. Nelsen, Social License to Operate, 2006.

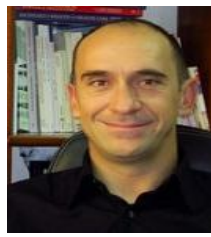

J. Andrés Domínguez-Gómez is assistant professor at the Department of Sociology, Social Work and Public Health (University of Huelva, Spain) from 1995. He got the $\mathrm{PhD}$ in sociology, the $\mathrm{MsC}$ in ergonomy and labour risks. He has collaborated in teaching and researching in several Universities and research institutions in different countries (Portugal, Brasil, Panama, Germany, the Netherlands or Finland, for instance). His main working-topics are socia impacts and risks assessment of development projects and methodologies for applied social research, which have centered his participation in 21 different private or public funded research projects (7 of them as Main Investigator) and several dozens of indexed publications (journal articles and books). Those topics and some others, such as social aspects of health, sociology of tourism, knowledge and technology transfer, have also led the activity of EISmethods LTD, funded by Prof. Dominguez-Gómez and partners as a spin-off company, associated with the University of Huelva.

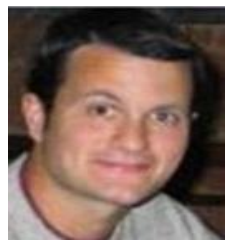

Fernando Relinque-Medina is associated professor a the Department of Sociology, Social Work and Public Health (University of Huelva, Spain) from 2015. He got the $\mathrm{MsC}$ in migrations and development. He is founder partner of EISmethods LTD, a spin-off company associated to University of Huelva. His teaching and researching activities are centered in social inequalities, vulnerable social groups and social impact assessment of development projects. He has published about ten journal articles and book chapters on such topics in the last three years. He has won different competitive public grants and excelence prizes on social enterpreneurship, and his closing PhD thesis has been developed on social dwelling and methodologies for proposing a new social-inclusive model in dwelling policies. 